\title{
Anthropomorphizing without Social Cues Requires the Basolateral Amygdala
}

\author{
Adam Waytz ${ }^{1}$, John T. Cacioppo ${ }^{2 *}$, Rene Hurlemann ${ }^{3}$, Fulvia Castelli ${ }^{3,4}$, \\ Ralph Adolphs ${ }^{5}$, and Lynn K. Paul ${ }^{5}$
}

\begin{abstract}
Anthropomorphism, the attribution of distinctively human mental characteristics to nonhuman animals and objects, illustrates the human propensity for extending social cognition beyond typical social targets. Yet, its processing components remain challenging to study because they are typically all engaged simultaneously. Across one pilot study and one focal study, we tested three rare people with basolateral amygdala lesions to dissociate two specific processing components: those triggered by attention to social cues (e.g., seeing a face) and those triggered by endogenous semantic knowledge (e.g., imbuing a
\end{abstract}

\section{INTRODUCTION}

Humans are undeniably social animals, exhibiting social behavior not merely toward other humans but also toward plants, animals, technological devices, and putative supernatural agents like God. This process of humanizing nonhumans, anthropomorphism, involves attributing distinctively human mental states (e.g., intentions, emotions, thoughts) to these entities (Epley, Waytz, \& Cacioppo, 2007). Anthropomorphism represents a fundamentally social process of attributing specifically mental characteristics (e.g., "my laptop is manipulative") rather than merely observable characteristics (e.g., "my laptop is silver"). However, the psychological processes that underlie anthropomorphism - and whose differential engagement may produce important individual differencesremain poorly understood. Here, we follow up on a prior study of the role of the amygdala in anthropomorphism (Heberlein \& Adolphs, 2004) to examine the differential engagement of bottom-up versus top-down processes in anthropomorphism.

Given the pervasiveness of anthropomorphism (Gray, Gray, \& Wegner, 2007), many have suggested that anthropomorphism represents a relatively automatic,

\footnotetext{
${ }^{1}$ Northwestern University, ${ }^{2}$ University of Chicago, ${ }^{3}$ University of Bonn, ${ }^{4}$ University of Pavia, ${ }^{5}$ California Institute of Technology *We dedicate this article to the memory of John Cacioppo, who passed away while the article was being finalized and whose seminal work in social neuroscience guided the questions and approaches we present here.
}

machine with animacy). A pilot study demonstrated that, like neurologically intact control group participants, the three amygdala-damaged participants produced anthropomorphic descriptions for highly socially salient stimuli but not for stimuli lacking clear social cues. A focal study found that the three amygdala participants could anthropomorphize animate and living entities normally, but anthropomorphized inanimate stimuli less than control participants. Our findings suggest that the amygdala contributes to how we anthropomorphize stimuli that are not explicitly social. effortless, and intuitive process. Humphrey (1976, p. 312) suggested that humans' tendency to reason socially about nonhuman stimuli represented "a predisposition among men to try to fit non-social material into a social mould" (emphasis ours). This view suggests that humans are naturally inclined to anthropomorphize. Similarly, Caporael (1986) suggested that anthropomorphism of machines is a "default schema," and later Caporael and Heyes (1997) proposed anthropomorphism as a "cognitive default." Guthrie (1997) similarly described religious belief in this way, stating "Our tendency to overestimate animacy and humanity in the world constitutes a cognitive default." These views suggest that we begin reasoning about nonhuman entities by anchoring on humanlike conceptions and adjusting from there (often insufficiently).

Some empirical evidence supports the view of anthropomorphism as automatic, effortless, and intuitive. Developmental research suggests children reason inductively about nonhuman animals through anthropocentrismusing "human" as the prototypical concept to attribute properties to nonhumans (Inagaki \& Hatano, 1987; Carey, 1985). Other work shows children as young as 12 months infer the mental states of simple shapes (Gergely, Nadasdy, Csibra, \& Biro, 1995), suggesting that anthropomorphism emerges early. Studies of adults also demonstrate people are biased to think of God anthropomorphically (Barrett \& Keil, 1996). Finally, a vast program of research on anthropomorphism of technology shows people "mindlessly" apply humanlike rules, norms, 
and stereotypes to interactions with computers (Nass \& Moon, 2000).

An alternate view suggests that anthropomorphism does not represent a cognitive default (Herrmann, Waxman, \& Medin, 2010) and must be first triggered to occur (Waytz, Klein, \& Epley, 2013). Considerable research has identified predictable stimulus-contingent triggers, such as the extent to which a particular stimulus moves or looks like a human. These human-like stimulus characteristics trigger people to access the concept of "human" to make inferences about a nonhuman stimulus. Research has also shown that different people anthropomorphize to varying degrees, with differing thresholds for doing so (Waytz, Cacioppo, \& Epley, 2010). For example, people are particularly prone to anthropomorphize when they desire social connection, seek to understand their environment, or encounter a stimulus that morphologically resembles a human (Epley, Waytz, Akalis, \& Cacioppo, 2008; Morewedge, Preston, \& Wegner, 2007; DiSalvo, Gemperle, Forlizzi, \& Kiesler, 2002; Eddy, Gallup, \& Povinelli, 1993). These findings suggest that anthropomorphism may be triggered by attention to stimulus-contingent social cues. However, the threshold for this trigger may also be influenced by the goals of the perceiver, thereby moderating the importance of stimulus-contingent features.

Research with clinical populations has also shown that psychiatric conditions or brain damage may influence the threshold at which anthropomorphism is triggered. For example, individuals with autism anthropomorphize shapes and computer-animated agents less than nonautistic individuals (Chaminade, Rosset, Da Fonseca, Hodgins, \& Deruelle, 2015; Castelli, Frith, Happé, \& Frith, 2002; Abell, Happe, \& Frith, 2000), whereas people with schizophrenia may overperceive human agency in the world around them (Gray, Jenkins, Heberlein, \& Wegner, 2011). Other research has shown that brain damage can reduce anthropomorphism, including damage to the right hemisphere (Weed, McGregor, Feldbaek Nielsen, Roepstorff, \& Frith, 2010) and amygdalae (Heberlein \& Adolphs, 2004).

The present research compares participants with specific amygdala lesions to neurologically healthy participants to partly dissociate the multiple psychological processes that guide anthropomorphism. Given the amygdala's role in attention and social cognition (see Adolphs, 2010) we hypothesized that amygdala damage would reduce anthropomorphism toward objects that normally engage goal-directed attention or that rely on semantic knowledge (such as a laptop computer, which does not look human). Such a dissociation revealed in neurological participants would help provide further insight into the architecture of anthropomorphizing and could even suggest biological markers associated with individual differences (such as amygdala size in healthy individuals).

A prior case study examining amygdala damage and anthropomorphism (Heberlein \& Adolphs, 2004) involved participants viewing and describing animated shapes designed to elicit anthropomorphic descriptions (Heider \& Simmel, 1944). In contrast to comparison participants (neurologically healthy individuals and individuals with damage to the OFC) who described the shapes anthropomorphically with mental states, the amygdala-damaged participant described the shapes in almost entirely inanimate terms (i.e., merely describing their motion). These findings suggested that amygdala damage could impair spontaneous anthropomorphism, supporting the amygdala's key role in social cognition. Importantly, however, when prompted with explicit questions that described the shapes as people with personalities, the amygdaladamaged participant provided anthropomorphic descriptions, showing that the ability, knowledge, and vocabulary to make social attributions were entirely intact. On a separate task, this participant was also able to describe a dog, an "overtly animate creature" (Heberlein \& Adolphs, 2004, p. 7489), in anthropomorphic terms.

These prior findings already demonstrated a striking dissociation between "spontaneous anthropomorphizing despite apparently intact social knowledge" (the title of the article of Heberlein \& Adolphs, 2004). However, limitations of that study raise important and unanswered questions that form the basis for the present work. First, the prior study (Heberlein \& Adolphs, 2004) was a singlecase study based on a single individual with amygdala lesions and based on a limited range of social stimuli. Perhaps most importantly, it did not systematically control for stimulus-contingent factors known to influence anthropomorphism. Consequently, those initial findings, intriguing as they are, leave open the question of what the specific factors might be and how they might depend on the amygdala. We took these prior findings to suggest a distinction between the capacity for mental state attribution and the perception of socially salient information triggering this attribution-essentially in a dissociation between social "performance" and social "competence" as processes that differentially depend on the amygdala. Based on these findings, we thus predicted that amygdaladamaged individuals will attribute mind (i.e., humanness) to entities that are sufficiently socially salient to trigger these attributions. We address this issue by examining whether amygdala lesions would impair anthropomorphic attributions made to explicitly social stimuli (i.e., stimuli that possess social cues, like facial features or animate motion) versus stimuli that totally lack visual social cues but may be associated with social knowledge through semantic knowledge and reasoning (e.g., a laptop computer). ${ }^{1}$

The present research examines this distinction further in light of research advances on both amygdala function and anthropomorphism. The amygdala's role in processing social and emotional information is well established (Martin \& Weisberg, 2003; Schultz et al., 2003), yet more recent views suggest the specific role of the amygdala in processing information relevant to the social and emotional 
"goals" and "motivations" of the perceiver (Cunningham \& Brosch, 2012; Sander, Grafman, \& Zalla, 2003). This motivational salience view suggests that the amygdala responds to both socially rewarding and socially threatening information (Van Bavel, Packer, \& Cunningham, 2008), as both are relevant to perceivers' goals to affiliate with trustworthy others and to avoid untrustworthy others. Also consistent with this hypothesis, research has demonstrated that bilateral amygdala damage is associated with reduced attention to socially relevant information such as others' eye gaze and facial expressions (Kennedy \& Adolphs, 2012), but that explicit instructions about where to attend can elicit relatively intact social judgments about such stimuli (even for amygdala-damaged individuals; Adolphs, Gosselin, et al., 2005). In light of this revised view of the amygdala's function, Heberlein and Adolphs' (2004) findings suggest the alternative explanation: The amygdala-damaged participant's reduced spontaneous anthropomorphism resulted not from an inability to anthropomorphize or process explicit social cues but rather from not perceiving these shapes as socially relevant or salient.

To gain additional neuroanatomical specificity to test this idea, we also tested neurological participants with more restricted lesions to the amygdala than the participant S. M. (original participant of Heberlein \& Adolphs, 2004). In particular, our three participants all had complete damage to the basolateral nucleus of the amygdala, but at most minor damage to the central or medial nuclei. The basolateral nucleus is the nucleus known to be bidirectionally connected with neocortex; thus, it receives visual inputs from visual neocortex and also sends feedback projections back to those visual cortices (Amaral, Price, Pitkanen, Carmichael, \& Aggleton, 1992). It is involved in attentional modulation of sensory information processing in these cortical regions. Indeed, tracer studies in monkeys show that the basolateral amygdala projects to all visual cortical regions, even those from which it does not receive inputs (Frees \& Amaral, 2005). It projects all the way back to primary visual cortex and is thus in a prime position, given this anatomical connectivity, to implement the function that we hypothesize to test here: providing a spontaneous attentional signal when socially relevant information is being processed, an attention signal that is normally automatically engaged and that triggers the retrieval of social knowledge and inferences used to make anthropomorphic attributions. The specific role for the basolateral amygdala in anthropomorphizing that we advance here is supported by several recent studies (Mormann et al., 2015; Spunt et al., 2015; Wang, Tudusciuc, et al., 2014; Wang, Xu, et al., 2014), some of them in the very same lesion participants we studied here.

Given the minimally social nature of the animated shapes in the prior study (Heberlein \& Adolphs, 2004), it is perhaps unsurprising that the amygdala-damaged participant did not spontaneously respond to them in a social manner. However, recent research on anthropomorphism has used a wider array of methods and a wider array of stimuli with varying social salience and relevance that may prompt anthropomorphism even for people with reduced sensitivity to social cues. For example, people attribute mental states to nonhuman stimuli such as plants that move at a humanlike speed compared with those that do not (Morewedge et al., 2007). People anthropomorphize robots that resemble humans more than robots that do not (DiSalvo et al., 2002), and people attribute more mental states to nonhuman animals that are more morphologically similar to humans (e.g., primates, dogs) than to those that are dissimilar (e.g., invertebrates, fish; Eddy et al., 1993). It is thus important to examine anthropomorphism across such a range of stimuli with varying degrees of explicitly social cues present.

The present studies use stimuli with varying degrees of social salience to examine the extent to which basolateral amygdala damage reduces anthropomorphism of nonhuman stimuli in general or whether such reductions in anthropomorphism are modulated by the presence of socially salient triggers (e.g., animacy). These stimuli are presented in both written and video format to demonstrate similar effects across media of varying visual intensity and to provide further generalizability of our findings.

\section{Overview of Studies}

Two studies examine our hypothesis. A pilot study with a limited set of healthy comparison participants tested whether amygdala-damaged participants would exhibit high versus low anthropomorphism toward stimuli prevalidated as displaying or not to displaying social cues (Abell et al., 2000; Castelli, Happé, Frith, \& Frith, 2000).

A focal study with a larger and better matched sample of healthy comparison participants tested whether amygdala-damaged participants (vs. healthy comparison participants) would differentially anthropomorphize stimuli that exhibit high versus low social salience. It showed that, whereas the participants with amygdala lesions could anthropomorphize stimuli with clear social cues present, they failed to do so when social cues were absent, revealing a dissociation in the processes that trigger anthropomorphizing.

\section{PILOT STUDY}

A pilot study tested whether amygdala-damaged participants are capable of anthropomorphism toward stimuli that convey clear social cues but not toward stimuli that lack these cues. Importantly, this study did so using stimuli that have already been validated as systematically varying in social salience (Abell et al., 2000; Castelli et al., 2000). We asked participants to describe video stimuli that convey low, moderate, or high degrees of social 
salience, with the hypothesis that they would anthropomorphize highly socially salient stimuli but not stimuli with low social salience.

\section{Methods}

\section{Participants}

Three women with bilateral lesions to the basolateral amygdala (Figure 1) caused by Urbach-Wiethe disease participated. All our amygdala participants had substantially more specific lesions than the original sole participant studied in Heberlein and Adolphs (2004), S. M., whose damage encompassed essentially the entire amygdala. Two of the participants, A. M. and B. G., are identical twin sisters from rural southern Germany, 36 years of age, married with children, and in full-time employment since receiving their 13 years of education in Germany. The third participant, A. P., is American. She is 27 years of age and has worked since she obtained her bachelor's degree. All three participants have an IQ in the average range, as measured by the Hamburg-Wechsler-Intelligenztest für Erwachsene Revision (A. M.: 101; B. G.: 96; Becker et al., 2012) or the Wechsler Abbreviated Scale of Intelligence (A. P.: 98; Wechsler, 1999), and are comparable to healthy comparison participants in this regard. Their lesions are all similarly symmetric and confined to the amygdala (A. M.: $1120 \mathrm{~mm}^{3}$ bilaterally; B. G.: $1150 \mathrm{~mm}^{3}$; A. P.: $710 \mathrm{~mm}^{3}$ ). The damage includes complete calcification of the basolateral amygdala with minor damage of other amygdaloid regions, including anterior and ventral cortical regions at the rostral level and lateral and medial parts of the central nucleus and amygdalohippocampal area at the caudal level.

We sought to maximize the generalizability of our findings by including all participants we could access who had bilateral amygdala lesions. Notably, we excluded those who had substantial damage outside the amygdala or who had cognitive impairments that would have precluded valid performances on our tasks. The only etiology known to produce relatively selective bilateral amygdala lesions is a genetic disease (Urbach-Wiethe disease, also called lipoid proteinosis), which is exceedingly rare (Hofer, 1973). Although there are a handful of other such participants in the world (e.g., Thornton et al., 2008), these other individuals typically have low IQ and additional cognitive impairments. The original amygdala participant in Heberlein and Adolphs (2004; participant S. M.) has also suffered additional medical problems in later years and has developed possible extra-amygdalar lesions, making her unsuitable for this study.

For a healthy group comparison, we utilized in this pilot study a small unpublished archival comparison sample (we were unable to obtain data from German speakers for the pilot study). Because the stimuli for this study are visual without use of text, primary language should have a limited impact on scores. The comparison sample was acquired from 14 individuals (3 women and 11 men) recruited from the community as part of an earlier, unrelated study. The comparison group and amygdala participants did not differ on age $(M=35.79$, $S D=15.89), t(15)=0.294, p=.773$, and Full Scale Intelligence Quotient $(M=105.86, S D=11.01), t(15)=$ $1.149, p=.268$. To maximize our comparison group size, we included all available comparison data, including data acquired from men (even though our amygdala participants are all women). ${ }^{2}$

\section{Materials}

The animation test The animation test consists of a set of 12 video clips (length: 34-45 sec) depicting two triangles moving against a framed white background. These clips have been used in prior research on social attributions to visual stimuli (Horan et al., 2009; Abell et al., 2000; Castelli et al., 2000). Half the animations depict also
Figure 1. T-1 weighted MRI images from participants with amygdala lesions. $\mathrm{A}=\mathrm{A}$. P.; $\mathrm{B}=\mathrm{A}$. M.; C $=$ B. G.

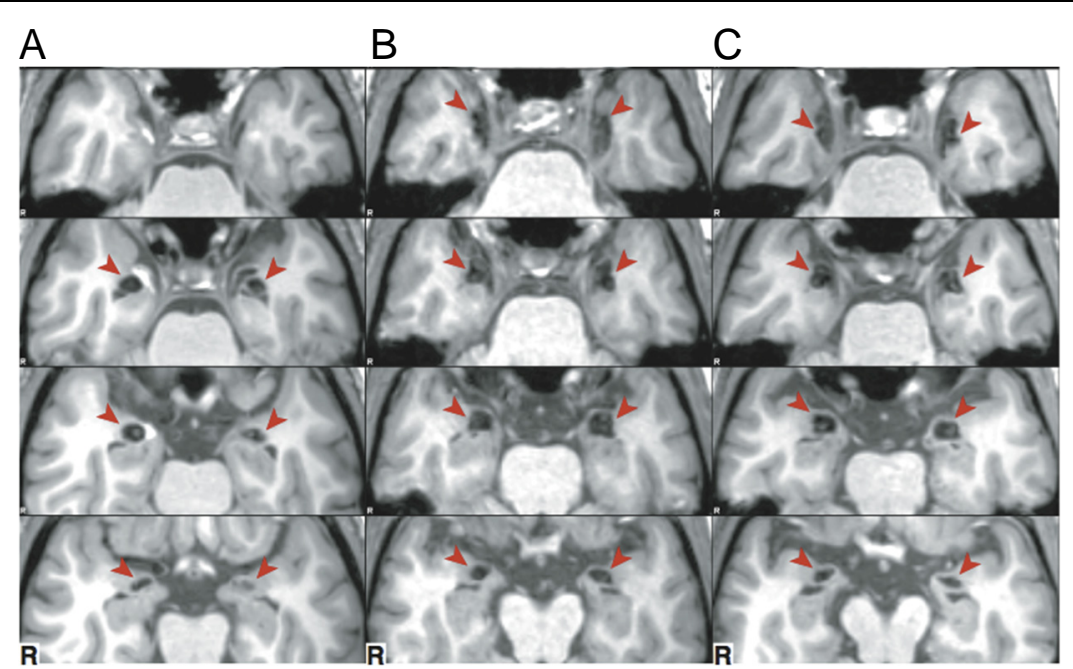


a framed enclosure with an opening. Three different types of interactions are compared, which are designed to convey differing levels of social cues: "theory of mind" and two control conditions: "goal-directed" and "random." The theory of mind animations in general feature one triangle pretending, persuading, seducing, or surprising another and thus convey a high degree of social cues (e.g., see https://www.youtube.com/watch? $\mathrm{v}=\mathrm{qH} 1 \mathrm{O} 616 \mathrm{FDq} 0)$. The four goal-directed animations show the two triangles dancing, chasing, fighting, or following and thus convey a moderate degree of social cues (e.g., see https://www.youtube.com/watch? $\mathrm{v}=\mathrm{dx} 7 \mathrm{MZh} 3 \mathrm{E} 3 \mathrm{vE})$. The four random animations depict the two triangles bouncing, drifting, spinning, or floating and thus convey a low degree of social cues, exhibiting more general properties of motion (e.g., see https://www.youtube.com/watch?v=57a_wbwEi5c). Across all animations, the shape, speed, and orientation of the triangles were kept as similar as possible.

\section{Procedure}

The animation test was presented to each participant individually, with stimulus order counterbalanced across participants. Instructions were presented on the computer screen and read aloud by the examiner. After viewing each animation, participants responded orally to the question, "What was happening in the animation?" All responses were recorded and later transcribed for scoring. The German-speaking participants with amygdala lesions responded orally in German, and responses were translated into English for scoring.

Scoring Deidentified answers were compiled subjectwise in random order and were evaluated by the test developer and creator of the scoring system, who was blind to participant diagnosis. Each item was scored along two dimensions: Anthropomorphism was measured through participants' "intentionality" score (as this score captures the degree of mental state attribution toward nonhuman stimuli, $0-5$ points) and "appropriateness" (0-3 points). Scoring was conducted using the methods described in Horan et al. (2009). Per this scoring system, high intentionality referred to description of complex, intentional mental states and purposeful movements related to the other character (triangle) whereas low intentionality referred to nondeliberate action such as "bouncing around," and high appropriateness referred to a clear answer identifying "the events depicted in the animations, as intended by the underlying scripts" whereas low appropriateness referred to no answer or stating, "I don't know" (Horan et al., 2009, p. 638). Statistical analyses were conducted using mean intentionality and appropriateness scores for each participant.

\section{Results and Discussion}

For all three levels (random, goal directed, and theory of mind), the amygdala-damaged participants' anthropomorphism (as measured by their intentionality score) and appropriateness ratings varied across the mean point of the comparison group and the confidence intervals (CIs) overlapped across groups (Figure 2). A 2 Group $\times$ 3 Animation level repeated-measures ANOVA was conducted for each rating (appropriateness and intentionality). Consistent with previous studies (Abell et al., 2000; Castelli et al., 2000), we found that anthropomorphism (i.e., intentionality) increased across the progression from random to goal directed to theory of mind in our sample, $F(1.222,18.325)=34.429, p<.001, \eta_{\mathrm{p}}^{2}=$ .697. In addressing comparison of amygdala participants and comparison participants, the groups did not differ on
Figure 2. Boxplots of mean intentionality and appropriateness ratings, with individual participant scores overlaid. ToM $=$ theory of mind.

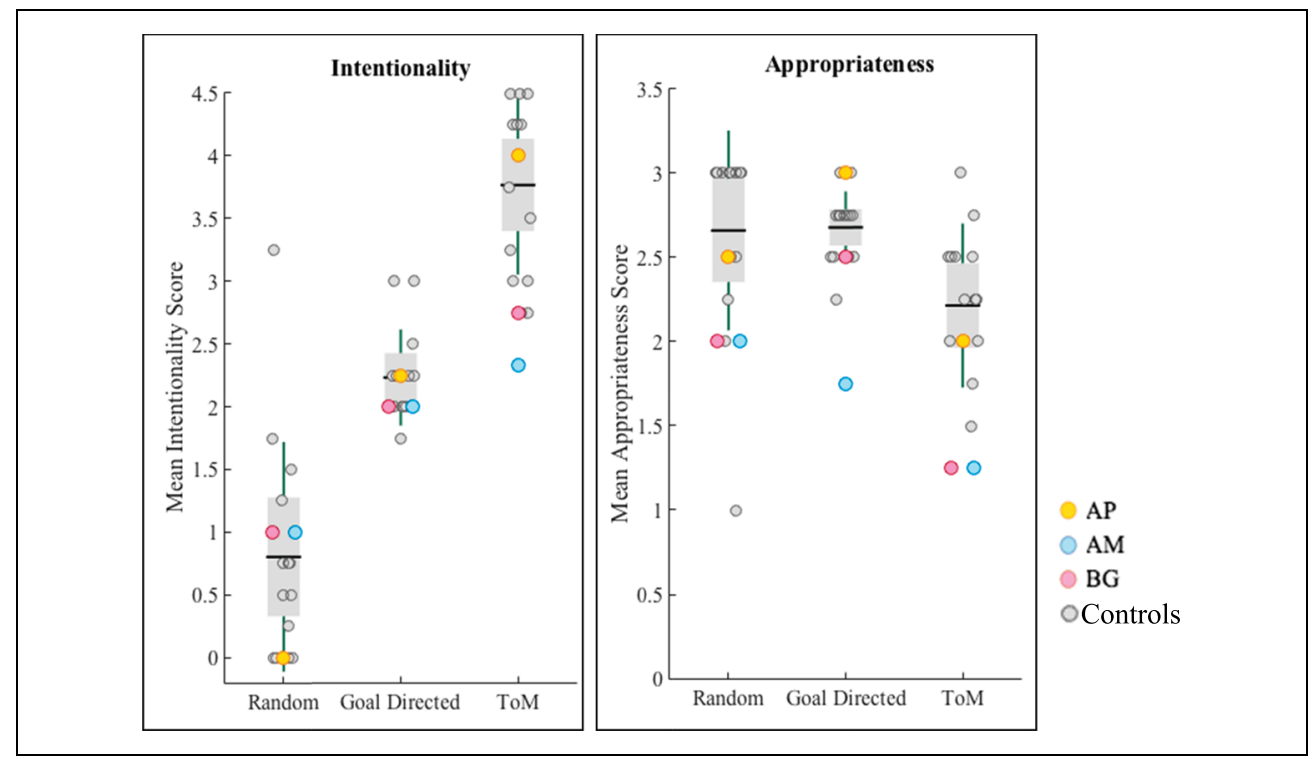


intentionality ratings overall, nor was there a significant interaction of group by animation level: Group, $F(1,15)=$ $2.236, p=.156, \eta_{\mathrm{p}}^{2}=.130$; Group $\times$ Animation Level, $F(1.222,18.325)=0.577, p=.490, \eta_{\mathrm{p}}^{2}=.037$. For appropriateness ratings, the overall pattern across conditions was also consistent with previous findings: Appropriateness ratings decreased across the progression from random to goal directed to theory of mind, $F(2,14)=9.702, p=.002, \eta_{\mathrm{p}}^{2}=.581$. However, although the groups did not differ on intentionality ratings, the amygdala-damaged participants' appropriateness ratings were generally lower than the comparison group, $F(1$, $15)=4.987, p=.041, \eta_{\mathrm{p}}^{2}=.250$, without a notable interaction of Group $\times$ Animation Level, $F(2,14)=$ $0.975, p=.402, \eta_{\mathrm{p}}^{2}=.122$. These results suggest the movement cues in these stimuli can elicit anthropomorphic descriptions from amygdala-damaged participants, but their descriptions may not accurately capture the specific social scripts commonly perceived in these stimuli.

This pilot study demonstrates that amygdala-damaged participants are capable of anthropomorphism toward stimuli that exhibit clear social cues. However, amygdaladamaged participants were less capable than healthy participants in accurately and appropriately describing the social interaction, despite accurately inferring specific mental states of distinct characters (e.g., Zaki, Bolger, \& Ochsner, 2008).

Both amygdala-damaged participants and healthy comparison participants exhibited similarly low levels of anthropomorphism toward clearly nonsocial stimuli. Rather than reflecting something about either group, this finding may simply reflect that these nonsocial (random) stimuli were insufficient to trigger anthropomorphic attributions from any observer, as they more clearly displayed nonsocial activities. Indeed, 4 of 15 comparison participants were at floor for anthropomorphism of these stimuli. Most critically, amygdala participants were capable of anthropomorphizing when stimuli clearly conveyed social salience.

This study differs from the prior work showing that an amygdala-damaged participant exhibited exceedingly low anthropomorphism toward animate video stimuli (similar to the one used in this study) compared with a healthy comparison group (Heberlein \& Adolphs, 2004). The development of the theory of mind videos used here indeed were inspired in part by Heider and Simmel's (1944) animation of shapes exhibiting contingent interaction that Heberlein and Adolphs (2004) employed in their study. Whereas amygdala-damaged participants here readily anthropomorphized shapes that displayed social cues, the amygdala-damaged participant in the 2004 study did not. This divergence may result from any number of methodological differences, including different location of amygdala damage across participant samples or differences in stimuli. Participants in this study viewed multiple videos clearly containing social cues in concert with other social videos clearly lacking in social relevance, as opposed to a single video in the 2004 study. It is possible that the present context enabled amygdala-damaged participants to anthropomorphize more. Importantly, the amygdala participant in the 2004 study, in a control task, did anthropomorphize a dog that conveyed clearly social cues, demonstrating that, as seen in the present research, animacy and social salience can produce anthropomorphism even for amygdala-damaged individuals. Most critically, this study demonstrates that amygdala-damaged individuals are more capable of anthropomorphism than previously thought and are capable of anthropomorphizing when social cues are clearly present.

\section{FOCAL STUDY}

\section{Methods}

\section{Participants}

As in the pilot study, we sought to maximize the generalizability of our findings by including all participants with bilateral amygdala lesions that we could access. Thus, we tested the same three amygdala participants as in the pilot study individually at Caltech using Qualtrics software.

The comparison group consisted of 93 total participants73 German-speaking healthy women, with mean age of 31.23 years $(S D=6.20$, range $=23-56$ years $)$, and 20 healthy American women, with mean age of 36.60 years $(S D=12.07$, range $=22-59$ years $)$. The amygdala-damaged participants and comparison participants were thus group matched for sex and were also group matched for age, $t(94)=-0.486, p=.628,95 \%$ CI $[-11.599,7.04]$. All comparison participants completed the study online and completed three measures described below.

We aimed to have 20 comparison participants for each amygdala-damaged participant (20 American, 40 German). In the end, our German colleague provided us access to 73 German participants. We used all available participants (3 amygdala, 20 American healthy comparisons, 73 German healthy comparisons) in our analyses and did not add or exclude any participants. We limited our sample to women because the available participants for our lesion group were all women, and we wanted to match our comparison group, which was larger than the pilot study, by sex. All data provided by the participants described here are analyzed below.

To quantify the difference between lesion participants and healthy comparison participants, we show effect sizes and used permutation testing to provide CIs. These ways of presenting data, in the absence of null hypothesis significance testing (NHST), are recommendations made broadly in psychology studies (Cumming, 2014). In select circumstances, we report results from NHST (specifically ANOVA). 


\section{Materials}

Individual differences in anthropomorphism questionnaire Participants completed the Individual Differences in Anthropomorphism Questionnaire (IDAQ; Waytz, Cacioppo, et al., 2010), a 30-item questionnaire that asks participants to indicate the extent to which technological entities (e.g., a television), natural entities (e.g., a mountain), and nonhuman animals (e.g., a cow) possess mental states (e.g., consciousness, emotions, intentions, free will, a mind of its own; $0=$ not at all, $10=$ very much). The 15 items that assess mental state attribution were summed to comprise the anthropomorphism subscale, and three subscale scores were calculated by summing five questions specific to each category $(\alpha=.82$ for technology, $\alpha=.80$ for animals, $\alpha=.85$ for nature). Fifteen additional items asked about the extent to which each item possesses a nonmental trait related to appearance or external behavior (e.g., active, lethargic, useful, good-looking, durable). These items were summed to constitute the nonanthropomorphism subscale, and three subscale scores were calculated by summing five questions specific to each category ( $\alpha=.26$ for technology, $\alpha=.37$ for animals, $\alpha=.50$ for nature). In the initial validation of the IDAQ, Waytz, Cacioppo, et al. (2010, pp. 221, 228) note that nonanthropomorphic items "are included to dissociate anthropomorphism from dispositional attribution more generally and to ensure that differences in anthropomorphism do not merely reflect differences in scale use...these items were developed simply to measure a diffuse set of nonanthropomorphic attributions rather than a single coherent construct." This is also why nonanthropomorphism composites for the IDAQ and for the stimuli described below have lower reliabilities than the anthropomorphism composites, which comprise items meant to represent a unified construct.

Dogs and robot videos Participants viewed two videos, one of two dogs and one of a mechanical robot that contained a computer screen and that moved on two wheels. In the dogs video, a smaller dog moves more variably and a larger dog moves more slowly and predictably - previous research has validated that these dogs vary on predictability (Epley et al., 2008), and they are described below as the unpredictable dog and predictable dog. After viewing each video, participants rated the extent to which each dog and the robot (described in the instructions as a "gadget") had mental states indicative of anthropomorphism: "a mind of its own," "free will," "consciousness," and the extent to which each stimulus "experienced emotions." These items were averaged to comprise an anthropomorphism score for each stimulus ( $\alpha \mathrm{s}=.83$ for both dogs, $\alpha=.89$ for the gadget). Participants also completed three items unrelated to anthropomorphism: the extent to which each stimulus was useful, durable, and good-looking - these items were averaged to comprise a nonanthropomorphism score for each stimulus $(\alpha=.70$ for the unpredictable dog, $\alpha=.71$ for the predictable dog, $\alpha=.59$ for the gadget). All items were rated on a 7 -point $(1=$ not at all, $7=$ very much) scale.

Predictable and unpredictable gadgets task Participants read descriptions of four technological gadgets: Clocky (a wheeled alarm clock that "runs away" so that you must get up to turn it off), CleverCharger (a battery charger designed to prevent overcharging), Pure Air (an air purifier for people with allergies or respiratory problems), and Pillow Mate (a torso-shaped pillow that can be programmed to give a "hug"). Participants evaluated each gadget described in both ways-as operating predictably or as operating unpredictably (previous research validated these descriptions as evoking predictability or unpredictability; Waytz, Morewedge, et al., 2010). After reading each description, participants answered a question about the extent to which they could control the gadget (not analyzed here) and completed five anthropomorphic mental-state ratings: the extent to which the gadget had "a mind of its own," had "intentions," had "free will," had "consciousness," and "experienced emotions." These items were averaged for all gadgets across each description type (predictable or unpredictable) to comprise an anthropomorphism score for each one (both $\alpha s=.95$ ). Participants also completed three items unrelated to anthropomorphism: the extent to which each device was attractive, efficient, and strongthese items were averaged for all gadgets across each description type (predictable or unpredictable) to comprise a nonanthropomorphism score for each one $(\alpha=$ .88 for unpredictable gadgets, $\alpha=.85$ for predictable gadgets). All items were rated on a 7 -point $(1=$ not at all, $7=$ very much) scale. Participants also completed short personality measures on loneliness, personal control, and familiarity with various stimuli for which we did not calculate scores or analyze because they did not pertain to the research question.

\section{Procedure}

Administration order of anthropomorphism measures was randomized across participants. For the German participants, all text was translated into German.

To control for differences in comparison group distributions for the two nationalities, we calculated $z$ scores for each amygdala participant's ratings relative to the culture-specific comparison group and for each comparison participant relative to the mean and standard deviation of all other comparison in that group.

We compared the lesion participants and comparison participants in two ways. First, we estimated how the three lesion participants would compare with three people randomly drawn from the general population. For each rating, we built a bootstrap population estimate 
Figure 3. Distribution of IDAQ scores from bootstrap samples (green $=$ anthropomorphism; blue $=$ nonanthropomorphism), with scores at the lower and upper bound of the $95 \% \mathrm{CI}$ shown by black dotted lines. Scores of individual amygdaladamaged participants are overlaid on top. *No overlap of CIs.

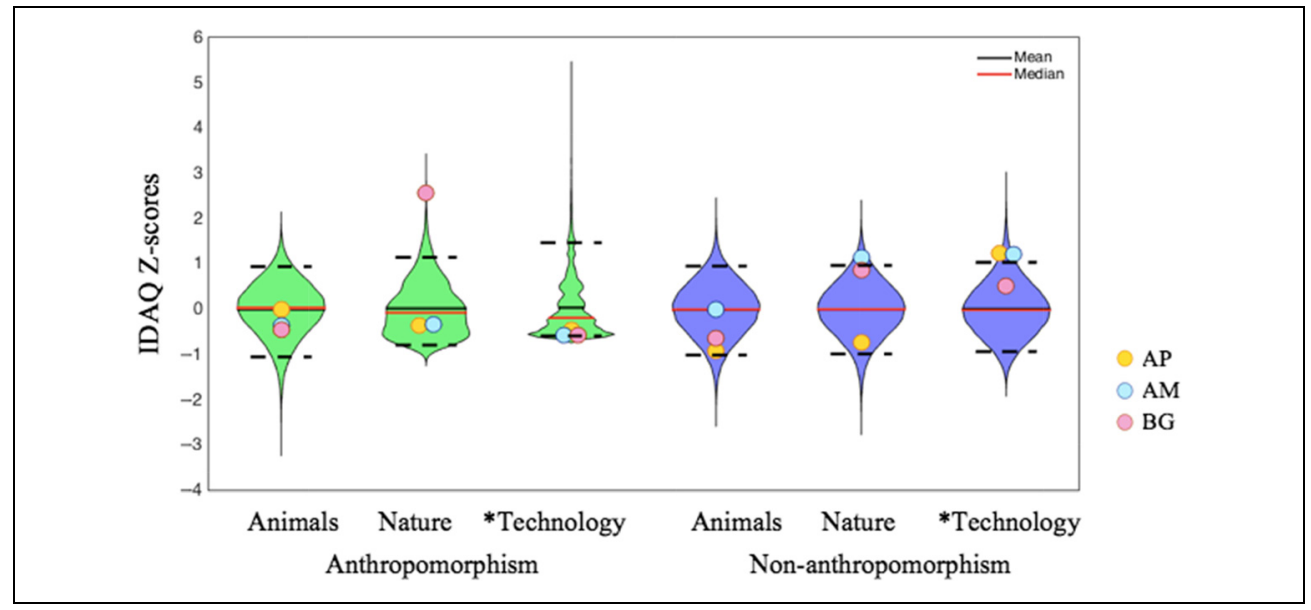

from 100,000 randomly selected (with replacement) groups of three comparison participants. For each measure of interest, the amygdala-damaged participants' scores were compared with the bootstrap-sampled distribution. Second, we compared CIs for each group (amygdala participants and original/nonbootstrapped comparison group) and reported the difference between each amygdala participant's score and the original comparison group mean (as a fraction of comparison group standard deviation).

\section{Results and Discussion}

Because of the small sample size among amygdaladamaged participants, we first consider variables that yielded clearly consistent results across all three amygdaladamaged participants. We will then comment on what we can learn from their pattern of results within each measure. We present results for anthropomorphism first and nonanthropomorphism second.

\section{Anthropomorphism Ratings}

Anthropomorphism ratings on all three tasks provided evidence that in general amygdala-damaged participants anthropomorphize stimuli with low social salience (inanimate technology-based stimuli, except the set of unpredictable gadgets that are known to trigger social attributions) to a lesser degree than the comparison group. This is evident when contrasting participants' ratings with the bootstrapped comparison distribution, as well as when contrasting with the original comparison group means. All three amygdala-damaged participants' anthropomorphism ratings were below the mean of the bootstrapped comparison distribution for technology (Figure 3), robots (Figure 4), and predictable gadgets (Figure 5). Table 1 reports differences between each participant's ratings and comparison group mean, along with CIs for both groups. To interpret the figures and table correctly, please note that Table 1 reports CIs from the actual comparison group whereas Figures 3-5 show distributions (and 95\% CIs) for the bootstrapped comparison group.

Compared with the comparison group mean rating for technology $(M=0.0388, S D=1.153)$, A. P.'s ratings were $0.42 S D$ lower and ratings for both A. M. and B. G. were 0.54 $S D$ lower. The amygdala lesion and comparison group averages were moderately distinct $(d=.50)$, with nonoverlapping 95\% CIs [-0.153, 0.329] for comparison participants and $[-0.578,-0.442]$ for amygdala participants.
Figure 4. Distribution of dogs and robot scores from bootstrap samples (green = anthropomorphism; blue = nonanthropomorphism), with scores at the lower and upper bound of the 95\% CI shown by black dotted lines. Scores of individual amygdala-damaged participants are overlaid on top. Predictable $=$ predictable dog; Unpredict. $=$ unpredictable dog. *No overlap of CIs.

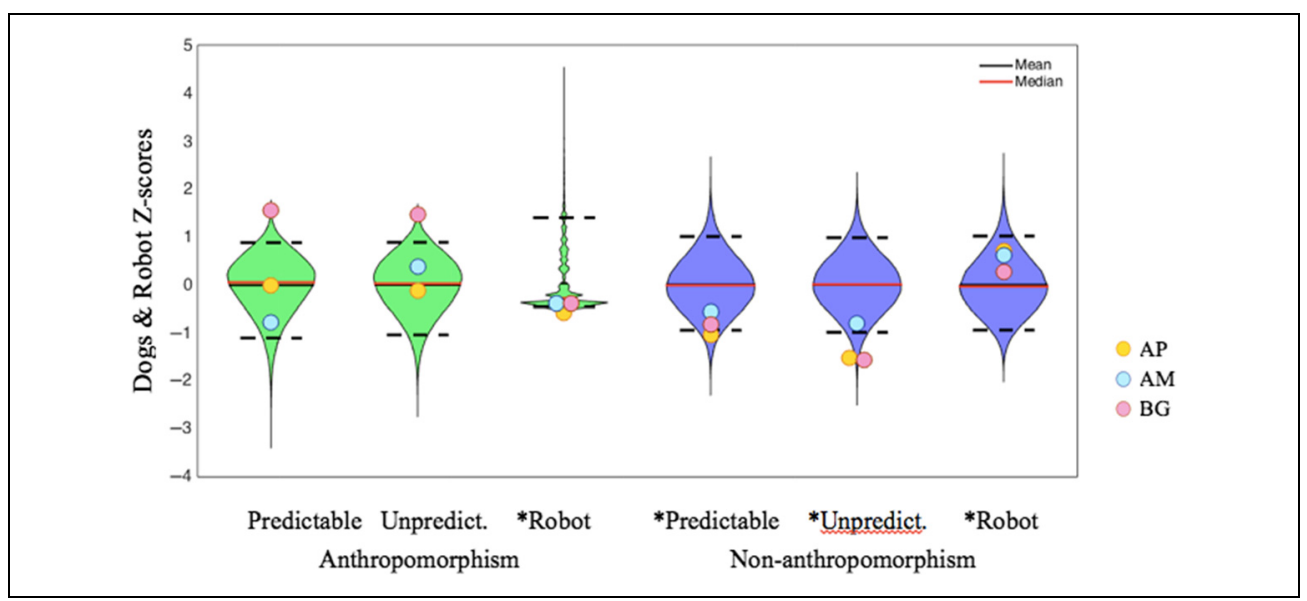


Figure 5. Distribution of predictable and unpredictable gadget scores from bootstrap samples (green $=$ anthropomorphism; blue = nonanthropomorphism), with scores at the lower and upper bound of the 95\% CI shown by black dotted lines. Scores of individual amygdala-damaged participants are overlaid on top. *No overlap of CIs.

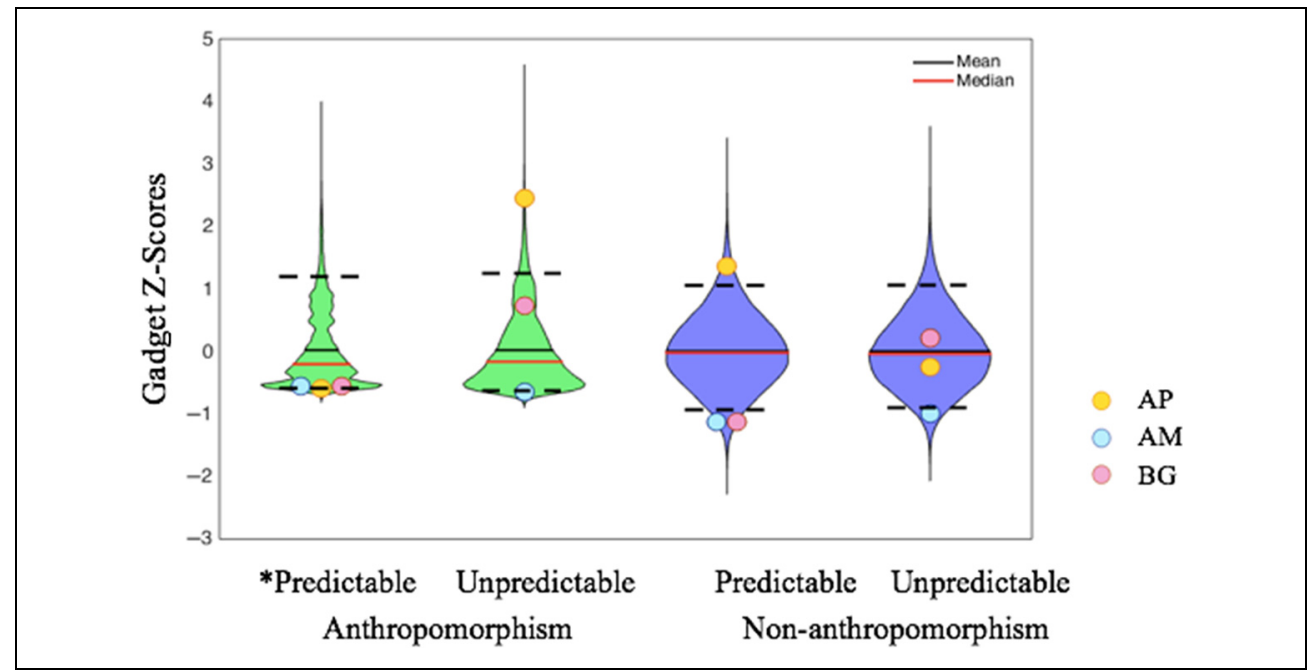

Compared with the bootstrap-sampled distribution, only $17.50 \%$ of the bootstrap comparison group had mean anthropomorphism ratings of technology below the mean of the amygdala participants.

Compared with the comparison group mean rating for robots $(M=0.032, S D=1.114)$, A. P.'s ratings were 0.55 $S D$ lower and ratings for both A. M. and B. G. were 0.37 $S D$ lower. The amygdala participant and comparison group averages were moderately distinct $(d=.43)$, with nonoverlapping $95 \%$ CIs $[-0.150,0.316]$ for comparison participants and $[-0.583,-0.379]$ for amygdala participants. Compared with the bootstrap-sampled distribution, only $17.67 \%$ of the bootstrap comparison group had mean anthropomorphism ratings of robots below the mean of the amygdala participants.

Compared with the comparison group mean rating for predictable gadgets $(M=0.030, S D=1.086)$, A. P.'s ratings were $0.56 S D$ lower and ratings for both $\mathrm{A}$. M. and B. G. were $0.53 S D$ lower. The amygdala participant and comparison group averages were moderately distinct $(d=.54)$, with nonoverlapping 95\% CIs [ $-0.162,0.290]$ for comparison participants and [-0.584, -0.545$]$ for amygdala participants. Compared with the bootstrapsampled distribution, only $8.2 \%$ of the bootstrap comparison group had mean anthropomorphism ratings of predictable gadgets below the mean of the amygdala participants.

For all other variables (IDAQ ratings of animals and nature, both dogs, and the unpredictable gadgets), the amygdala-damaged participants' anthropomorphism ratings varied across the mean point of the comparison group and the CIs were overlapping across groups (Table 1). Thus, these results are not discussed here.

\section{Nonanthropomorphism Ratings}

On nonanthropomorphism ratings, the amygdaladamaged participants gave consistently higher ratings to technology (Figure 3) and robots (Figure 4) and consistently lower ratings to both predictable and unpredictable dogs (Figure 4) compared with the bootstrapped comparison distribution. For all other variables, the amygdaladamaged participants' nonanthropomorphism ratings varied across the mean point of the comparison group and the CIs were overlapping across groups (Table 1). As with anthropomorphism ratings that overlapped across groups, these results are not discussed here.

Collapsing over comparison participants, associations between anthropomorphism and nonanthropomorphism ratings ranged from nonsignificantly correlated, $r(91)=$ $.189, p=.07$ (for the robot), to moderately strongly correlated, $r(91)=.53, p<.001$ (for the unpredictable gadgets), with an average correlation size of .38 (including amygdala participants reveals the same pattern of results with an average correlation size of .37). Thus, anthropomorphism and nonanthropomorphism appear moderately positively associated (but not redundant), and in no cases were anthropomorphism and nonanthropomorphism negatively correlated. This finding is consistent with previous work demonstrating that nonanthropomorphic attribution is independent from anthropomorphic attribution (Waytz, Cacioppo, et al., 2010).

In the amygdala-damaged group, anthropomorphism and nonanthropomorphism scores on the IDAQ were not significantly correlated (with $d f=1$, animals $r=$ .043 , nature $r=.374$, technology $r=.521$ ). Although also not significant, anthropomorphism and nonanthropomorphism ratings were negatively correlated on the predictable and unpredictable dogs $(r=-.383, r=$ $-.256)$ and robot $(r=-.654$, again with $d f=1)$. Finally, the correlation of anthropomorphism and nonanthropomorphism ratings for unpredictable gadgets was positive but nonsignificant $(r=.561)$, but there was a strong negative correlation for predictable gadgets, $r(1)=-1.00, p<.001$. 
Table 1. Comparison of Anthropomorphism Ratings from Amygdala Participants and Comparison Sample

\begin{tabular}{|c|c|c|c|c|c|c|c|c|}
\hline & \multirow{2}{*}{$\begin{array}{l}\text { Bootstrapped Comparison } \\
\text { Samples with Mean above } \\
\text { Amygdala Participants (\%) }\end{array}$} & \multicolumn{4}{|c|}{$95 \%$ CI (Z scores) } & \multicolumn{3}{|c|}{$\begin{array}{l}\text { Fraction of SD Different } \\
\text { from Comparison Mean }\end{array}$} \\
\hline & & \multicolumn{2}{|c|}{ Comparisons } & \multicolumn{2}{|c|}{ Amygdala } & A. $P$. & A. $M$. & B. $G$. \\
\hline \multicolumn{9}{|l|}{ Anthropomorphism Ratings } \\
\hline \multicolumn{9}{|l|}{ IDAQ } \\
\hline Animals & 66.06 & -0.227 & 0.197 & -0.553 & -0.046 & -0.01 & -0.10 & -0.52 \\
\hline Nature & 16.49 & -0.176 & 0.254 & -0.350 & 2.576 & -0.35 & -0.34 & 2.43 \\
\hline Technology ${ }^{\mathrm{a}}$ & 82.50 & -0.153 & 0.329 & -0.578 & -0.442 & -0.42 & -0.54 & -0.54 \\
\hline \multicolumn{9}{|l|}{ Dogs and robot } \\
\hline Predictable dog & 35.87 & -0.239 & 0.188 & -0.787 & 1.555 & 0.00 & -0.74 & 1.49 \\
\hline Unpredictable dog & 16.46 & -0.224 & 0.195 & 0.048 & 1.469 & -0.11 & 0.38 & 1.43 \\
\hline $\operatorname{Robot}^{\mathrm{a}}$ & 82.33 & -0.150 & 0.316 & -0.583 & -0.379 & -0.55 & -0.37 & -0.37 \\
\hline \multicolumn{9}{|l|}{ Gadgets } \\
\hline Predictable gadgets $^{\mathrm{a}}$ & 91.80 & -0.162 & 0.290 & -0.584 & -0.545 & -0.56 & -0.53 & -0.53 \\
\hline Unpredictable gadgets & 12.51 & -0.161 & 0.295 & -0.187 & 2.455 & 2.22 & -0.61 & 0.63 \\
\hline \multicolumn{9}{|c|}{ Nonanthropomorphism Ratings } \\
\hline \multicolumn{9}{|l|}{ IDAQ } \\
\hline Animals & 80.05 & -0.217 & 0.202 & -0.817 & -0.006 & -0.88 & 0.00 & -0.61 \\
\hline Nature & 24.11 & -0.216 & 0.205 & -0.741 & 1.046 & -0.71 & 1.10 & 0.83 \\
\hline Technology ${ }^{\mathrm{b}}$ & 5.82 & -0.198 & 0.221 & 0.501 & 1.223 & 1.18 & 1.16 & 0.48 \\
\hline \multicolumn{9}{|l|}{ Dogs and robot } \\
\hline Predictable dog ${ }^{\mathrm{a}}$ & 91.72 & -0.202 & 0.219 & -1.040 & -0.570 & -1.00 & -0.55 & -0.80 \\
\hline Unpredictable dog ${ }^{\mathrm{a}}$ & 98.56 & -0.207 & 0.212 & -1.541 & -0.799 & -1.46 & -0.77 & -1.50 \\
\hline $\operatorname{Robot}^{\mathrm{b}}$ & 18.58 & -0.200 & 0.221 & 0.275 & 0.678 & 0.68 & 0.60 & 0.26 \\
\hline \multicolumn{9}{|l|}{ Gadgets } \\
\hline Predictable gadgets & 68.28 & -0.198 & 0.229 & -1.129 & 1.368 & 1.29 & -1.08 & -1.08 \\
\hline Unpredictable gadgets & 69.68 & -0.195 & 0.226 & -0.986 & 0.059 & -0.24 & -0.95 & 0.20 \\
\hline
\end{tabular}

${ }^{\mathrm{a} A m y g d a l a}$ group below comparison group with no overlap of CIs.

${ }^{\mathrm{b}}$ Amygdala group above comparison group with no overlap of CIs.

For most stimuli, the relationship between anthropomorphism and nonanthropomorphism ratings was similar in amygdala-damaged participants and comparison participants, with two exceptions; 2 (Group: healthy comparison vs. amygdala participants) $\times 2$ (Attribution: anthropomorphic vs. nonanthropomorphic) ANOVAs conducted with each stimulus revealed significant interaction effects for IDAQ technology, $F(1$, $94)=3.793, p=.044, \eta_{\mathrm{p}}^{2}=.042$, and the unpredictable $\operatorname{dog}, F(1,94)=8.756, p=.004, \eta_{\mathrm{p}}^{2}=.085$. Both are cases where amygdala-damaged participants score lower than healthy comparison participants on anthropomorphic ratings and higher on nonanthropomorphic ratings.
This finding raises the possibility that social and nonsocial attribution operate in somewhat of a zero-sum fashion for these individuals on these particular stimuli, such that when an entity lacks social relevance (such as technology) people become more attuned to alternate features such as its efficiency or appearance. However, we hesitate to speculate too much on this finding because it does not emerge for all stimuli (and may be a function of our stimuli), but it is worth exploring in future research.

Response patterns within the IDAQ and dogs and robot tasks indicate both comparison participants and amygdala-damaged participants anthropomorphize 
inanimate entities (technology) to a lesser degree than animate entities (animals). This was true of both comparison groups (German and American) separately. Although amygdala-damaged participants appear to anthropomorphize inanimate entities to an even lesser degree than comparison participants, Gadgets task results suggest that cues to animacy-in this case, unpredictability - may markedly increase their anthropomorphism of technology. These findings support our interpretation that animacy is a primary trigger for anthropomorphism and are consistent with other work linking animacy to mind attribution (Wheatley, Weinberg, Looser, Moran, \& Hajcak, 2011; Looser \& Wheatley, 2010).

Finally, although both the comparison participants and amygdala-damaged participants distinguish between animals and technology by anthropomorphizing the former more than the latter, this pattern does not emerge for nonanthropomorphic attribution. These findings suggest that amygdala-damaged participants' distinction between animate and inanimate entities for anthropomorphism is specific to this type of social attribution and does not apply to nonsocial attribution more generally.

\section{GENERAL DISCUSSION}

This study argues that damage to the basolateral amygdala diminishes mental state attribution toward nonhuman entities, but only when these entities lack explicit social cues. Our findings thus provide substantial improvements and further insight over prior neuroscience studies on anthropomorphizing: We include three neurological participants (unlike the single one in Heberlein \& Adolphs, 2004), who have specific damage to only a set of subnuclei within the amygdala (thought to subserve spontaneous attentional functions) and use a much more comprehensive set of stimuli and tasks to better specify how the amygdala contributes to anthropomorphism. The pilot study showed equivalent levels of anthropomorphism between amygdala-damaged participants and comparison participants across stimuli but critically showed that amygdala participants anthropomorphized stimuli with explicit social cues to a greater degree than stimuli lacking these cues.

Central to our research question, we measured anthropomorphism and nonanthropomorphic attribution toward a variety of stimuli, for both amygdala-damaged participants and healthy comparison participants. The focal study showed that amygdala-damaged participants appear to anthropomorphize animate, living entities such as animals and natural life forms to an equivalent and, in some cases, greater degree than do the comparison participants. Yet, amygdala-damaged participants did not tend to anthropomorphize inanimate entities such as technology.

This novel dissociation supports the interpretation that there are multiple routes to anthropomorphizing. One route depends on attention triggered by explicit social cues, such as an entity's animate movement, and this processing component does not depend on the basolateral amygdala. A second route depends on attention triggered by other cues that are not explicitly social but that can become associated with social meaning; this second processing component requires the basolateral amygdala. Our findings thus utilized the dissociation afforded by basolateral amygdala damage to reveal two distinct processing components to anthropomorphizing.

The present findings may seem surprising in light of prior work that has demonstrated impaired processing of social stimuli such as faces in participants with amygdala damage-yet, these were in fact the class of stimuli producing the most normal responses from our participants with amygdala lesions. We argue that these data suggest a revision to the widely held view that the amygdala is disproportionately important for social perception. That view is not so much wrong as insufficiently detailed, since it conflates the multiple processes that typically contribute to social perception: The amygdala is critical only for some of these and not others. Specifically, processes that can be triggered by overt, explicit social cues do not require the amygdala, whereas those that depend on learned associations of cues with semantic or contextual social knowledge do. Examples of the former would be explicitly social stimuli, which are anthropomorphized relatively normally in our amygdala participants. Prior studies in such participants have similarly shown that processes like conscious detection of fear faces (Tsuchiya, Moradi, Felsen, Yamazaki, \& Adolphs, 2009) or change detection of people and animals (Wang, Tsuchiya, New, Hurlemann, \& Adolphs, 2015) are normal. By contrast, examples of the latter class of processes would be anthropomorphizing an air purifier, which offers no explicit social cues and the social attributes of which must instead be inferred entirely from semantic knowledge. This class of stimuli is anthropomorphized less by our amygdala participants compared with healthy participants. Prior studies are also consistent with this finding, when reinterpreted according to our hypothesis. The well-known deficits in recognizing emotions (Adolphs, Gosselin, et al., 2005; Adolphs, Tranel, \& Baron-Cohen, 2002; Adolphs et al., 1999) and other social information (Adolphs, Tranel, \& Damasio, 1998) from faces in participants with amygdala lesions depend on judgments that require considerable and nuanced semantic knowledge retrieved from relatively impoverished cues (e.g., the degree of each emotion the person shown in the stimulus might feel or how trustworthy somebody might be judged just from static photos of their face with no other information provided) and disappear when additional explicit disambiguating social cues are present (e.g., body postures). Another study found that participants with amygdala lesions were impaired in their aesthetic judgments for completely nonsocial and abstract visual stimuli (Adolphs \& Tranel, 1999), bearing similarity to the 
present deficit for nonsocial stimuli like an air purifier: In both cases, it is the retrieval of learned associations with these stimuli that appears to depend on the amygdala.

Perhaps the most congruent prior study (conducted in the exact same three individuals with amygdala lesions) is one by Harrison, Hurlemann, and Adolphs (2015), which found that these participants' judgments of others' approachability were the most abnormal when they looked at pictures of people whose faces had been erased; that study concluded that the amygdala's role must extend beyond stimulus-triggered processing. Consistent with our interpretation in the present article, this work supports the view that the basolateral amygdala is not necessary for triggering social inferences in response to explicit social cues but rather for triggering social inferences when social cues are absent or ambiguous, and the inferences instead have to be guided by context, semantic knowledge, and reasoning. Indeed, the distinction that our finding supports may have even broader application. For instance, amygdala lesions impair aspects of emotional memory, but here again it seems to be the more semantically and abstract components of a memory ("gist") rather than its concrete features ("details") that is impaired (Adolphs, Buchanan, \& Tranel, 2005).

\section{Limitations of the Present Work}

Our findings are strongly constrained by the participants available. In the case of the participants with amygdala lesions, we have only three, and there are substantial differences between them, making it difficult to generalize conclusions about the consequences of amygdala damage. For instance, one was American, and the other two were German; education and age differed (college or not; 27 vs. 36 years). Even for the two German twins, prior studies have shown discordant brain activations to social stimuli (Becker et al., 2012). In addition, another limitation of the current sample is that two of the three amygdala-damaged participants are identical twins (and are thus genetically identical, with highly similar childhood experiences) and thus are not fully independent observations. Future studies corroborating the present conclusions will likely need to come from different types of studies, such as fMRI studies examining amygdala activation in healthy individuals where larger sample sizes are possible.

A second limitation concerns the dependency of responses on the tasks on context and culture. Future studies should include large-sample (e.g., online) studies that explicitly examine the effects of age, sex, and aspects of cultural background to help further understand the sensitivity of anthropomorphizing to such possibly confounding factors.

Finally, it is important to reiterate the logic of human lesion studies. Our findings cannot demonstrate that the amygdala directly causes anthropomorphizing; they only show that the process can be disrupted in the absence of the amygdala. This leaves open the exact causal contribution that the amygdala makes in healthy individuals. It is also critical to remember that all patients had amygdala lesions that are disproportionate to the basolateral amygdala and that this damage was sustained developmentally. These facts leave open the possibility that other components of the amygdala might play additional, or different, functional roles in anthropomorphizing, and they leave open the possibility that acute amygdala lesions could produce substantially more severe impairments in anthropomorphizing since they would preclude possible compensation due to plasticity and reorganization throughout the lives of our participants.

\section{Relationship to Other Studies}

Expanding on an initial finding by Heberlein and Adolphs (2004) using one focal stimulus and one amygdaladamaged participant, our study suggests that the relationship between amygdala function and anthropomorphism is more nuanced. Our fundamental interpretation that basolateral amygdala damage reveals two distinct processing components to anthropomorphizing is supported by additional studies on the basolateral amygdala using electrophysiology or fMRI. One recent study showed that single neurons recorded from the human basolateral amygdala encode information based on holistic processing and experience, such as the identity of a person, but not information based on single social features, such as the direction of eye gaze (Mormann et al., 2015). Relatedly, as we noted above, other work found individuals with lesions to the basolateral amygdala to have normal visual attention to explicitly social stimuli (pictures of people) in visual search (Wang, Xu, et al., 2014). Both of these studies argue that the long-recognized role of the basolateral amygdala in attention is more nuanced than previously thought: It appears to be based not on lowlevel visual cues but rather on high-level information. This reinterpretation was strengthened in another recent finding that found neurons recorded in the human basolateral amygdala in fact respond not merely to the features in faces that make them show a particular emotion (happiness and fear in that study) but rather to the subjective judgment that the participant makes about those faces (even when they mistake a fear face for a happy face, for instance; Wang, Tudusciuc, et al., 2014). Like this study, these existing studies of the amygdala argue for a role in processing associations between stimuli and their social meaning.

One additional study is particularly important, given that it used the same individuals with basolateral amygdala lesions that we used in our present study. Participants were asked to engage in theory of mind tasks while undergoing functional neuroimaging (Spunt et al., 2015). Not only were the amygdala participants able to perform the tasks, but they activated an entirely normal 
cortical network that is normally activated when people engaged in theory of mind. This study corroborates the finding that social attribution in an explicit task does not require the amygdala and is consistent with the finding here that participants with amygdala lesions are able to make explicit social attributions and have normal social knowledge.

Another notable finding from our present study that relates to prior work on the amygdala concerns the amygdala-damaged participants' evaluations of technology we found. The only case in which some amygdaladamaged participants anthropomorphized technology to a greater degree than healthy comparison participants was when evaluating technology that operated unpredictably - a condition that makes gadgets appear more human-like (Waytz, Morewedge, et al., 2010; Epley et al., 2007). This additional finding may also be related to other work showing that the amygdala is particularly sensitive to encoding the unpredictability of stimuli and presumably engaging attentional processes as a consequence (Herry et al., 2007; Whalen, 1999).

The present research differs from prior work on amygdala damage and anthropomorphism not only in using a richer set stimuli but also in using only explicit questions about anthropomorphism in the focal study rather than measuring spontaneous anthropomorphism alone (Heberlein \& Adolphs, 2004). Use of explicit questions may have prompted participants to consider anthropomorphic features. However, this would only increase the chance of false-negative findings (i.e., prompting more anthropomorphism than participants would naturally produce) and if anything would reduce the chance for false positives. Indeed, for stimuli lacking social salience, even explicit prompting did not encourage anthropomorphism in our amygdala participants in the focal study. The pilot study, which measured spontaneous anthropomorphism alone, mimicked this pattern of results.

Another critical difference between this study and prior work was our inclusion of specific nonanthropomorphic measures to provide an important dissociation from the anthropomorphic measures. As seen with comparison participants, amygdala-damaged participants' ratings of anthropomorphic characteristics (items pertaining to mental states) overall showed a different pattern of attribution from nonanthropomorphic characteristics (items pertaining to behavior or appearance). Thus, for these participants, anthropomorphism appears to be dissociable from trait attribution more generally.

\section{Conclusions}

In summary, basolateral amygdala damage reduces anthropomorphic attribution toward purely nonsocial stimuli but does not alter anthropomorphism toward nonhuman stimuli with social cues. Thus, socially relevant stimuli that act or look humanlike, or that prompt a motivation for social sense-making, evoke anthropomorphism and mental state reasoning for people with amygdala damage. However, when evaluating stimuli that do not possess socially relevant features, people with amygdala damage may not anthropomorphize, suggesting they require more explicit contextual cues to engage the process. This result is consistent with the work showing that amygdala damage does not compromise performance on an explicit false-belief reasoning task, which prompts individuals to consider the mind of another person (Spunt et al., 2015), as well as work in monkeys with bilateral amygdala lesions suggesting that the basic repertoire of social behavior is intact even though context does not elicit it spontaneously (Amaral et al., 2003). These findings support the view that the amygdala is not essential for anthropomorphism per se but rather that it is essential for engaging in anthropomorphism as a function of the goal relevance and social salience provided by a particular context or instruction.

Beyond merely providing insight into amygdala function, these findings suggest a critical distinction between two processing components of social cognition. The first, which appears compromised as a result of basolateral amygdala damage, is the rapid and immediate detection of a stimulus' social relevance, when contextual cues are limited. The second, which is not compromised by amygdala damage, is the attribution of social-in the present research, mental-characteristics when the stimulus is unambiguously (explicitly) social or when there are sufficient context cues to elicit a social interpretation. Here, anthropomorphism emerges even for participants with amygdala lesions, but at a higher threshold than what is normally seen, suggesting that many forms of anthropomorphism are not automatic but rather rely on explicit knowledge that a stimulus should be considered social. The attribution of mental states to a stimulus merely requires the possession of social knowledge (i.e., general knowledge about mental state capacities), and this does not depend on the amygdala. But attentional and motivational processes need to be engaged first to trigger the retrieval of such social knowledge, and this is what is impaired in our amygdala participants.

\section{Acknowledgments}

German comparison participants were recruited by Tania Singer. R. H. was supported by the German Research Foundation (DFG) BE 5465/2-1 and HU 1302/4-1. R. A. and L. K. P. were supported by a Conte Center from NIMH (P50MH094258).

Reprint requests should be sent to Adam Waytz, Kellogg School of Management, Northwestern University, 2211 Campus Dr 5175 , Evanston, IL 60208, or via e-mail: a-waytz@kellogg.northwestern. edu.

\section{Notes}

1. Although we describe the processes of social cuetriggered anthropomorphism triggered and semantic-driven 
anthropomorphism as separate, we speculate that these processes operate on a spectrum. For example, anthropomorphizing an animal that looks and acts like a human (e.g., a chimpanzee) likely relies more on social cues and less on semantic knowledge, whereas anthropomorphizing a computer that is inanimate and lacks observable humanlike features likely relies more on semantic knowledge because it elicits fewer social cues. In this way, the distinction between these two processes represents a standard distinction in cognition between bottom-up (social cue-triggered) versus top-down (semantic-driven) processing, simply applied to anthropomorphism.

2. We believe this sex imbalance between comparison groups is unlikely to affect results given evidence suggesting that anthropomorphism does not differ by sex per se (Saxton, Mackey, McCarty, \& Neave, 2016; Severson \& Lemm, 2016).

\section{REFERENCES}

Abell, F., Happe, F., \& Frith, U. (2000). Do triangles play tricks? Attribution of mental states to animated shapes in normal and abnormal development. Cognitive Development, 15, $1-16$.

Adolphs, R. (2010). What does the amygdala contribute to social cognition? Annals of the New York Academy of Sciences, 1191, 42-61.

Adolphs, R., Buchanan, T. W., \& Tranel, D. (2005). Amygdala damage impairs memory for gist but not details of complex stimuli. Nature Neuroscience, 8, 512-519.

Adolphs, R., Gosselin, F., Buchanan, T., Tranel, D., Schyns, P., \& Damasio, A. (2005). A mechanism for impaired fear recognition after amygdala damage. Nature, 433, 68-72.

Adolphs, R., \& Tranel, D. (1999). Preferences for visual stimuli following amygdala damage. Journal of Cognitive Neuroscience, 11, 610-616.

Adolphs, R., Tranel, D., \& Baron-Cohen, S. (2002). Amygdala damage impairs recognition of social emotions from facial expressions. Journal of Cognitive Neuroscience, 14, 1264-1274.

Adolphs, R., Tranel, D., \& Damasio, A. R. (1998). The human amygdala in social judgment. Nature, 393, 470-474.

Adolphs, R., Tranel, D., Hamann, S., Young, A., Calder, A., Anderson, A., et al. (1999). Recognition of facial emotion in nine subjects with bilateral amygdala damage. Neuropsychologia, 37, 1111-1117.

Amaral, D., Capitanio, J., Jourdain, M., Mason, W., Mendoza, S., \& Prather, M. (2003). The amygdala: Is it an essential component of the neural network for social cognition? Neuropsychologia, 41, 235-240.

Amaral, D., Price, J., Pitkanen, A., Carmichael, S., \& Aggleton, J. (1992). Anatomical organization of the primate amygdaloid complex. In The amygdala: Neurobiological aspects of emotion, memory, and mental dysfunction (pp. 1-66).

Barrett, J. L., \& Keil, F. C. (1996). Conceptualizing a nonnatural entity: Anthropomorphism in God concepts. Cognitive Psychology, 31, 219-247.

Becker, B., Mihov, Y., Scheele, D., Kendrick, K. M., Feinstein, J. S., Matusch, A., et al. (2012). Fear processing and social networking in the absence of a functional amygdala. Biological Psychiatry, 72, 70-77.

Caporael, L. R. (1986). Anthropomorphism and mechanomorphism: Two faces of the human machine. Computers in Human Behavior, 2, 215-234.

Caporael, L. R., \& Heyes, C. (1997). Why anthropomorphize? Folk psychology and other stories. In R. W. Mitchell, N. S. Thompson, \& H. L. Miles (Eds.), Anthropomorphism, anecdotes, and animals (pp. 59-73). Albany, NY: State University of New York Press.

Carey, S. (1985). Conceptual change in childhood. Cambridge, MA: MIT Press.

Castelli, F., Frith, C., Happé, F., \& Frith, U. (2002). Autism, Asperger syndrome and brain mechanisms for the attribution of mental states to animated shapes. Brain, 125, 1839-1849.

Castelli, F., Happé, F., Frith, U., \& Frith, C. (2000). Movement and mind: A functional imaging study of perception and interpretation of complex intentional movement patterns. Neuroimage, 12, 314-325.

Chaminade, T., Rosset, D., Da Fonseca, D., Hodgins, J. K., \& Deruelle, C. (2015). Anthropomorphic bias found in typically developing children is not found in children with autistic spectrum disorder. Autism, 19, 248-251.

Cumming, G. (2014). The new statistics. Psychological Science, 25, 7-29.

Cunningham, W. A., \& Brosch, T. (2012). Motivational salience amygdala tuning from traits, needs, values, and goals. Current Directions in Psychological Science, 21, 54-59.

DiSalvo, C. F., Gemperle, F., Forlizzi, J., \& Kiesler, S. (2002). All robots are not created equal: The design and perception of humanoid robot heads. Paper presented at the 4th Conference on Designing Interactive Systems: Processes, Practices, Methods, and Techniques.

Eddy, T. J., Gallup, G. G., \& Povinelli, D. J. (1993). Attribution of cognitive states to animals: Anthropomorphism in comparative perspective. Journal of Social Issues, 49, 87-101.

Epley, N., Waytz, A., Akalis, S., \& Cacioppo, J. T. (2008). When we need a human: Motivational determinants of anthropomorphism. Social Cognition, 26, 143-155.

Epley, N., Waytz, A., \& Cacioppo, J. T. (2007). On seeing human: A three-factor theory of anthropomorphism. Psychological Review, 114, 864-886.

Frees, J., \& Amaral, D. G. (2005). The organization of projections from the amygdala to visual cortical areas TE and V1 in the macaque monkey. The Journal of Comparative Neurology, 486, 295-317.

Gergely, G., Nadasdy, Z., Csibra, G., \& Biro, S. (1995). Taking the intentional stance at 12 months of age. Cognition, 56 , $165-193$.

Gray, H. M., Gray, K., \& Wegner, D. M. (2007). Dimensions of mind perception. Science, 315, 619.

Gray, K., Jenkins, A. C., Heberlein, A. S., \& Wegner, D. M. (2011). Distortions of mind perception in psychopathology. Proceedings of the National Academy of Sciences, U.S.A., 108, 477-479.

Guthrie, S. E. (1997). Anthropomorphism: A definition and a theory. In R. W. Mitchell, N. S. Thompson, \& H. L. Miles (Eds.), Anthropomorphism, anecdotes, and animals (pp. 50-58). Albany: State University of New York Press.

Harrison, L. A., Hurlemann, R., \& Adolphs, R. (2015). An enhanced default approach bias following amygdala lesions in humans. Psychological Science, 26, 1543-1555.

Heberlein, A., \& Adolphs, R. (2004). Impaired spontaneous anthropomorphizing despite intact social knowledge and perception. Proceedings of the National Academy of Sciences, U.S.A., 101, 7487-7491.

Heider, F., \& Simmel, M. (1944). An experimental study of apparent behavior. American Journal of Psychology, 57, 243-259.

Herrmann, P., Waxman, S. R., \& Medin, D. L. (2010). Anthropocentrism is not the first step in children's reasoning about the natural world. Proceedings of the National Academy of Sciences, U.S.A., 107, 9979-9984.

Herry, C., Bach, D. R., Esposito, F., Di Salle, F., Perrig, W. J., Scheffler, K., et al. (2007). Processing of temporal 
unpredictability in human and animal amygdala. Journal of Neuroscience, 27, 5958-5966.

Hofer, P. (1973). Urbach-Wiethe disease: A review. Acta Dermato-venereologico, 53, 5-52.

Horan, W. P., Nuechterlein, K. H., Wynn, J. K., Lee, J., Castelli, F., \& Green, M. F. (2009). Disturbances in the spontaneous attribution of social meaning in schizophrenia. Psychological Medicine, 39, 635-643.

Humphrey, N. K. (1976). The social function of intellect. In P. P. G. Bateson \& R. A. Hinde (Eds.), Growing points in ethology (pp. 303-317). Cambridge: Cambridge University Press.

Inagaki, K., \& Hatano, G. (1987). Young children's spontaneous personification as analogy. Child Development, 58 , 1013-1020.

Kennedy, D. P., \& Adolphs, R. (2012). The social brain in psychiatric and neurological disorders. Trends in Cognitive Sciences, 16, 559-572.

Looser, C. E., \& Wheatley, T. (2010). The tipping point of animacy. How, when, and where we perceive life in a face. Psychological Science, 21, 1854-1862.

Martin, A., \& Weisberg, J. (2003). Neural foundations for understanding social and mechanical concepts. Cognitive Neuropsychology, 20, 575-587.

Morewedge, C. K., Preston, J., \& Wegner, D. M. (2007). Timescale bias in the attribution of mind. Journal of Personality and Social Psychology, 93, 1-11.

Mormann, F., Niediek, J., Tudusciuc, O., Quesada, C. M., Coenen, V. A., Elger, C. E., et al. (2015). Neurons in the human amygdala encode face identity, but not gaze direction. Nature Neuroscience, 18, 1568-1570.

Nass, C., \& Moon, Y. (2000). Machines and mindlessness: Social responses to computers. Journal of Social Issues, 56, 81-103.

Sander, D., Grafman, J., \& Zalla, T. (2003). The human amygdala: An evolved system for relevance detection. Reviews in the Neurosciences, 14, 303-316.

Saxton, T. K., Mackey, L. L., McCarty, K., \& Neave, N. (2016). A lover or a fighter? Opposing sexual selection pressures on men's vocal pitch and facial hair. Behavioral Ecology, 27, 512-519

Schultz, R. T., Grelotti, D. J., Klin, A., Kleinman, J., Van der Gaag, C., Marois, R., et al. (2003). The role of the fusiform face area in social cognition: Implications for the pathobiology of autism. Philosophical Transactions of the Royal Society of London, Series B, Biological Sciences, 358, 415-427.

Severson, R. L., \& Lemm, K. M. (2016). Kids see human too: Adapting an individual differences measure of anthropomorphism for a child sample. Journal of Cognition and Development, 17, 122-141.

Spunt, R. P., Elison, J. T., Dufour, N., Hurlemann, R., Saxe, R., \& Adolphs, R. (2015). Amygdala lesions do not compromise the cortical network for false-belief reasoning. Proceedings of the National Academy of Sciences, U.S.A., 112, 4827-4832.

Thornton, H. B., Nel, D., Thornton, D., van Honk, J., Baker, G. A., \& Stein, D. J. (2008). The neuropsychiatry and neuropsychology of lipoid proteinosis. The Journal of Neuropsychiatry and Clinical Neurosciences, 20, 86-92.

Tsuchiya, N., Moradi, F., Felsen, C., Yamazaki, M., \& Adolphs, R. (2009). Intact rapid detection of fearful faces in the absence of the amygdala. Nature Neuroscience, 12, 1224.

Van Bavel, J. J., Packer, D. J., \& Cunningham, W. A. (2008). The neural substrates of in-group bias a functional magnetic resonance imaging investigation. Psychological Science, 19, $1131-1139$

Wang, S., Tsuchiya, N., New, J., Hurlemann, R., \& Adolphs, R. (2015). Preferential attention to animals and people is independent of the amygdala. Social Cognitive and Affective Neuroscience, 10, 371-380.

Wang, S., Tudusciuc, O., Mamelak, A. N., Ross, I. B., Adolphs, R., \& Rutishauser, U. (2014). Neurons in the human amygdala selective for perceived emotion. Proceedings of the National Academy of Sciences, U.S.A., 111, E3110-E3119.

Wang, S., Xu, J., Jiang, M., Zhao, Q., Hurlemann, R., \& Adolphs, R. (2014). Autism spectrum disorder, but not amygdala lesions, impairs social attention in visual search. Neuropsychologia, 63, 259-274.

Waytz, A., Cacioppo, J. T., \& Epley, N. (2010). Who sees human?: The stability and importance of individual differences in anthropomorphism. Perspectives on Psychological Science, 5, 219-232.

Waytz, A., Klein, N., \& Epley, N. (2013). Imagining other minds: Hair triggered but not hare brained. New York: Oxford University Press.

Waytz, A., Morewedge, C. K., Epley, N., Monteleone, G., Gao, J.-H., \& Cacioppo, J. T. (2010). Making sense by making sentient: Effectance motivation increases anthropomorphism. Journal of Personality and Social Psychology, 99, 410-435.

Wechsler, D. (1999). WASI administration and scoring manual. San Antonio, TX: Psychological Corporation.

Weed, E., McGregor, W., Feldbaek Nielsen, J., Roepstorff, A., \& Frith, U. (2010). Theory of Mind in adults with right hemisphere damage: What's the story? Brain and Language, $113,65-72$.

Whalen, P. (1999). Fear, vigilance, and ambiguity: Initial neuroimaging studies of the human amygdala. Current Directions in Psychological Science, 7, 177-187.

Wheatley, T., Weinberg, A., Looser, C., Moran, T., \& Hajcak, G. (2011). Mind perception: Real but not artificial faces sustain neural activity beyond the N170/NPP. PLoS One, 6, e17960.

Zaki, J., Bolger, N., \& Ochsner, K. (2008). It takes two: The interpersonal nature of empathic accuracy. Psychological Science, 19, 399-404. 